\title{
Two-step pressure-induced collapse of magnetic order in the MnGe chiral magnet
}

\author{
M. Deutsch, ${ }^{1}$ O. L. Makarova, ${ }^{1,2}$ T. C. Hansen, ${ }^{3}$ M. T. Fernandez-Diaz ${ }^{3}$ V. A. Sidorov, ${ }^{4}$ A. V. Tsvyashchenko, ${ }^{4,5}$ \\ L. N. Fomicheva, ${ }^{4}$ F. Porcher, ${ }^{1}$ S. Petit, ${ }^{1}$ K. Koepernik, ${ }^{6}$ U. K. Rößler, ${ }^{6}$ and I. Mirebeau ${ }^{1, *}$ \\ ${ }^{1}$ CEA, Centre de Saclay, DSM/IRAMIS/Laboratoire Léon Brillouin, F-91191 Gif-sur-Yvette, France \\ ${ }^{2}$ National Research Center “Kurchatov Institute," 123182 Moscow, Russia \\ ${ }^{3}$ Institut Laue Langevin, 6 rue Jules Horowitz, BP 156, F-38042 Grenoble, France \\ ${ }^{4}$ Vereshchagin Institute for High Pressure Physics, Russian Academy of Sciences, 142190, Troitsk, Moscow, Russia \\ ${ }^{5}$ Skobeltsyn Institute of Nuclear Physics, MSU, Vorobevy Gory 1/2, 119991 Moscow, Russia \\ ${ }^{6}$ Leibniz Institute for Solid State and Material Research IFW, D-01069 Dresden, Germany
}

(Received 6 March 2014; published 15 May 2014)

\begin{abstract}
Cubic helimagnets such as MnSi and FeGe have provided paradigmatic cases of pressure-induced collapse of band ferromagnetism in metals, accompanied with exotic partial order and chiral spin textures. Isostructural MnGe stands out owing to its much shorter helix pitch and high magnetic moment. By combining resistivity, ac susceptibility, and neutron diffraction measurements under very high pressure, we show that the helical order in MnGe transforms around $6 \mathrm{GPa}$ from a high-spin to a low-spin state, recalling the weak ferromagnetism of $\mathrm{MnSi}$ at ambient pressure. Helical order collapses only above $10 \mathrm{GPa}$. The spin-state transition is supported by ab initio calculations.
\end{abstract}

DOI: 10.1103/PhysRevB.89.180407

PACS number(s): 75.30.Kz, 71.10.Hf, 75.25.-j

The family of intermetallic cubic compounds with the acentric cubic B20 structure has been a laboratory of intriguing properties. The chiral helimagnets $\mathrm{MnSi}$ and $\mathrm{FeGe}$, and the anomalous paramagnet FeSi have been extensively investigated for a long time in fundamental studies on magnetism, transport, and quantum phase transitions [1-3]. B20 metals show long-period helical structures, and vortexlike spin textures or skyrmions [4,5], which can be controlled by an electric current, inducing a topological Hall effect (THE) with possible applications in spintronics [6,7].

Quantum phase transitions (QPTs) are of fundamental interest not only in solid state physics, but also in studies of critical phenomena, Bose-Einstein condensate or cold atoms. They occur at zero temperature by varying some control parameter such as pressure or chemical composition. B20 metals such as $\mathrm{MnSi}$ or $\mathrm{FeGe}$ provide textbook examples of pressure-induced QPTs. Pressure changes the electronic structure, inducing new effects when magnetism collapses. In $\mathrm{MnSi}$, pressure greatly contributes to reveal its original magnetism by enhancing the stability of the skyrmion phase and the THE [8,9] and inducing a QPT towards a non-Fermi liquid (NFL) state with partial magnetic order and slow dynamics. The low critical pressure $\left(P_{C} \sim 1.4 \mathrm{GPa}\right)$ enables a fine microscopic description of the NFL phase, emerging under pressure without true quantum criticality $[3,10]$.

Germanides with a B20 structure have stronger ferromagnetic properties than the silicides $[11,12]$, as required to exploit their chiral magnetism. MnGe shows the strongest ferromagnetism among the B20 metals [13], together with giant THE and Nernst effects [14,15]. These effects, still not fully explained, make MnGe a great candidate for spintronics applications. In this Rapid Communication, we show that applying very high pressures reveals a unique character of MnGe magnetism, namely, a two-step pressure-induced

*isabelle.mirebeau@cea.fr collapse of the magnetic order. We attribute this anomalous sequence of quantum transitions to its peculiar band structure. The interplay of spin orbit coupling (SOC) and a quantum phase transition as found here for $\mathrm{MnGe}$ is also important in other fields of physics, such as spinor Bose condensation in cold atomic gases [16], where artificial gauge fields mimic the SOC perturbation.

MnGe was studied only recently due to its difficult synthesis. Its ordering temperature $T_{N}=170(5) \mathrm{K}$ and ordered Mn moment of about $2 \mu_{B}[14,17]$ are well above the values in $\mathrm{MnSi}\left(29 \mathrm{~K}\right.$ and $\left.0.4 \mu_{B}\right)$. The chiral order coincides with a small structural distortion [17]. The incommensurate wave vector $\mathbf{k}=(0,0, \zeta)$ parallel to a $(001)$ axis levels off at a value of 0.167 below $30 \mathrm{~K}$. It corresponds to the shortest helix pitch in the B20 series ( $29 \AA$ instead of $180 \AA$ in $\mathrm{MnSi}$ or $700 \AA$ in FeGe). A skyrmion lattice state was postulated based on resistivity and small angle neutron scattering on powder samples [14,18], but remains to be firmly established. High pressure experiments still need to be conducted, but $a b$ initio calculations have predicted the band structure to evolve under pressure from strong band ferromagnetism via a half-metallic state towards a collapse of spin polarization [19]. Experimental tests of this prediction are difficult, as the expected pressure range is above $10 \mathrm{GPa}$. Our study of MnGe under pressure combines susceptibility, resistivity up to $5.6 \mathrm{GPa}$, and neutron diffraction up $10.1 \mathrm{GPa}$, allowing a microscopic characterization of the evolution of magnetism. The pressure phase diagram shows a magnetic transition around $6 \mathrm{GPa}$, and the chiral order persists with a low ordered Mn moment above $10 \mathrm{GPa}$. Refined $a b$ initio calculations identify the first transition as the result of a pressure-induced high-spin (HS) to low-spin (LS) transition. We associate this very peculiar QPT with the onset of an intermediate fluctuating magnetoelastic state, specific to $\mathrm{MnGe}$.

Polycrystalline MnGe was synthesized and the magnetic susceptibility and electric resistivity were measured [see the Supplemental Material (SM) [20]]. At ambient pressure the ac susceptibility shows a broad peak around $T_{N}=180(5) \mathrm{K}$ and 

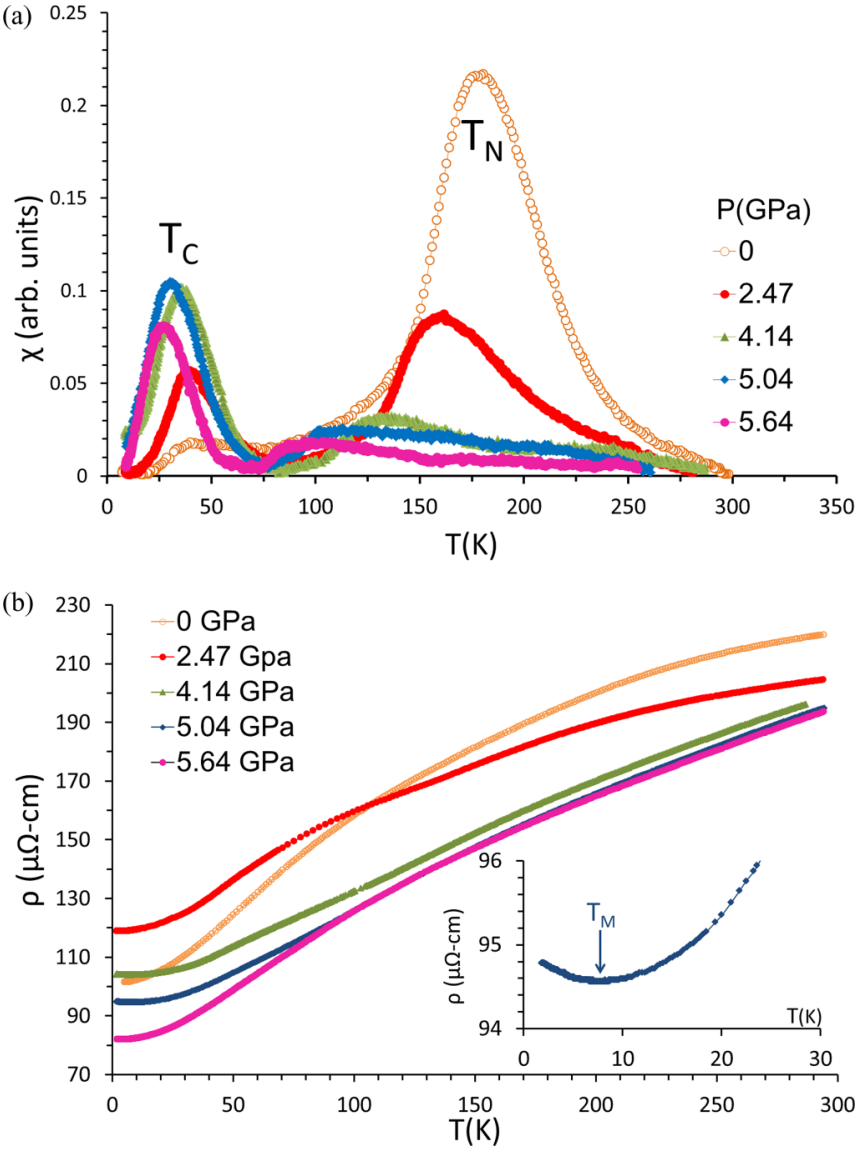

FIG. 1. (Color online) (a) MnGe susceptibility vs temperature at several pressures. (b) MnGe resistivity vs temperature at several pressures.

another weaker anomaly at a lower temperature, $T_{C}=40(3) \mathrm{K}$ [Fig. 1(a)]. Under pressure, the upper peak shifts down and transforms into a broad asymmetric bump. Concomitantly the weaker anomaly at $T_{C}$ transforms into a pronounced peak and its position shifts downwards. At $5.64 \mathrm{GPa}$ the peak intensities and positions of both peaks decrease and the upper transition becomes ill defined. The transition at $T_{C}$ coincides with a saturation of the helix pitch (see the SM) and with irreversibilities of the magnetization depending on cooling conditions [21]. Both transitions are revealed by broad peak line shapes, suggesting crossover regions in the phase diagram. The lower transition at $T_{C}$ likely reflects a blocking of helical domain motion when the modulation pitch tends to lock in. No anomaly was observed at $T_{N}$ or $T_{C}$ in the specific heat, which agrees with broad crossover regions around $T_{N}$ and $T_{C}$.

At ambient pressure, the resistivity shows standard metallic behavior [Fig. 1(b)], saturating at low temperature, as expected by defect scattering of the electrons. A weak anomaly is observed around $T_{N}$. More importantly, the temperature dependence of the resistivity shows an upturn at $T_{M}<T_{C}<T_{N}$ in the pressure range 2-5.6 GPa [see the inset of Fig. 1(b)]. This suggests a remarkable change in the band structure associated with a weak localization below $T_{M}$ and is a precursor to a more profound change at higher pressure. The phase diagram deduced from the susceptibility and resistivity is discussed below in comparison with neutron data.

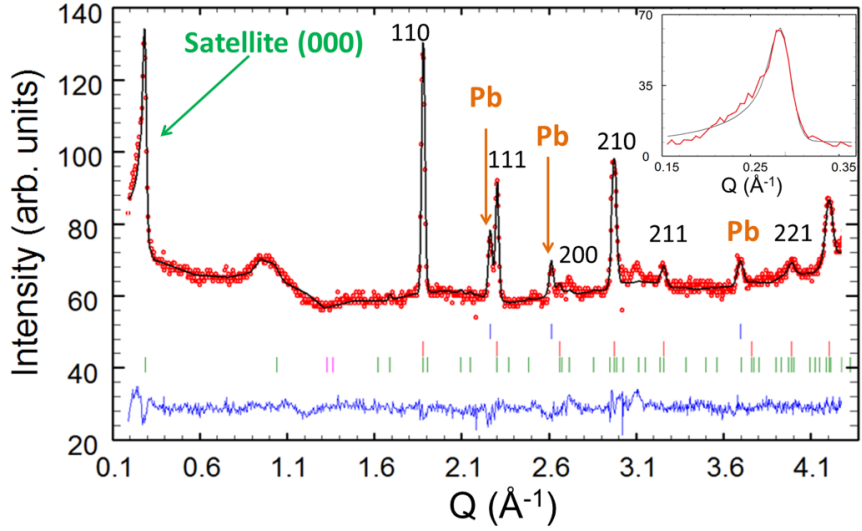

FIG. 2. (Color online) Diffraction pattern obtained with a D20 diffractometer at $20 \mathrm{~K}$ and $4.3 \mathrm{GPa}$. The solid black line is a FULLPROF refinement; tick marks indicate Bragg peak positions for the $\mathrm{Pb}$ and MnGe crystal phases, and for the MnGe magnetic phase, respectively. The solid blue line is the difference between calculated and measured patterns. The inset shows a zero satellite after subtraction of the nonmagnetic background at $190 \mathrm{~K}$ (red curve) and its fit (black curve).

We performed two sets of high pressure neutron diffraction experiments on D20 and G6.1 diffractometers, respectively (see the SM). A typical pattern measured on D20 at $20 \mathrm{~K}$ and 4.3 GPa is shown in Fig. 2. The magnetic pattern was obtained by subtracting a pattern well above $T_{N}$ (typically $190 \mathrm{~K}$ at 1.9 and $4.3 \mathrm{GPa}, 130 \mathrm{~K}$ at $6.6 \mathrm{GPa}$, and $80 \mathrm{~K}$ for the highest pressures to keep the same background conditions). The intense zero satellite with a strong asymmetric shape is clearly seen at low angles. Its position and intensity (calibrated to that of the nuclear peaks) are directly related to the helix pitch and ordered Mn moment, respectively. We refined the $\mathrm{MnGe}$ crystal phase in the $P 2{ }_{1} 3$ cubic space group, and assumed the same type of magnetic order as at ambient pressure (see the details in the SM). The zero satellite is indexed with an incommensurate wave vector $\mathbf{k}=(0,0, \zeta)$ in a reciprocal lattice unit, where the helix pitch $\Lambda=a / \zeta$. We focus here on its pressure dependence at low temperature. Figure 3 shows the evolution of the ground-state ordered moment and helix pitch. At low temperature, the satellite width is limited by the instrumental resolution, which means that long range ordered (LRO) helical domains are stabilized with a typical size above $1000 \AA$. With increasing pressure, a substantial change of the helix pitch from the ground-state value $\Lambda \simeq 29 \AA$ at ambient pressure to a shorter pitch of $19 \AA$ under pressure is found along with a substantial reduction of the ordered Mn moment under pressure, where it saturates for $p>6 \mathrm{GPa}$ to a reduced value of about $0.7 \mu_{B}$.

We determine $T_{N}$ from the temperature dependence of the Mn moment deduced from the neutron data (see the SM). Figure 4 reports the characteristic temperatures $T_{N}, T_{C}$, and $T_{M}$ as determined by neutrons, susceptibility, and resistivity versus pressure. The resulting phase diagram below $T_{N}$ is divided into two pressure regions. The gray bar around $6 \mathrm{GPa}$ marks the transformation between these regions. In the low pressure region the magnetic moment and helix pitch are larger and both decrease with pressure. Beyond this pressure, constant low moment and helix pitch are found which persist up to $T_{N}$. At all pressures, the ordered Mn moment remains below the 

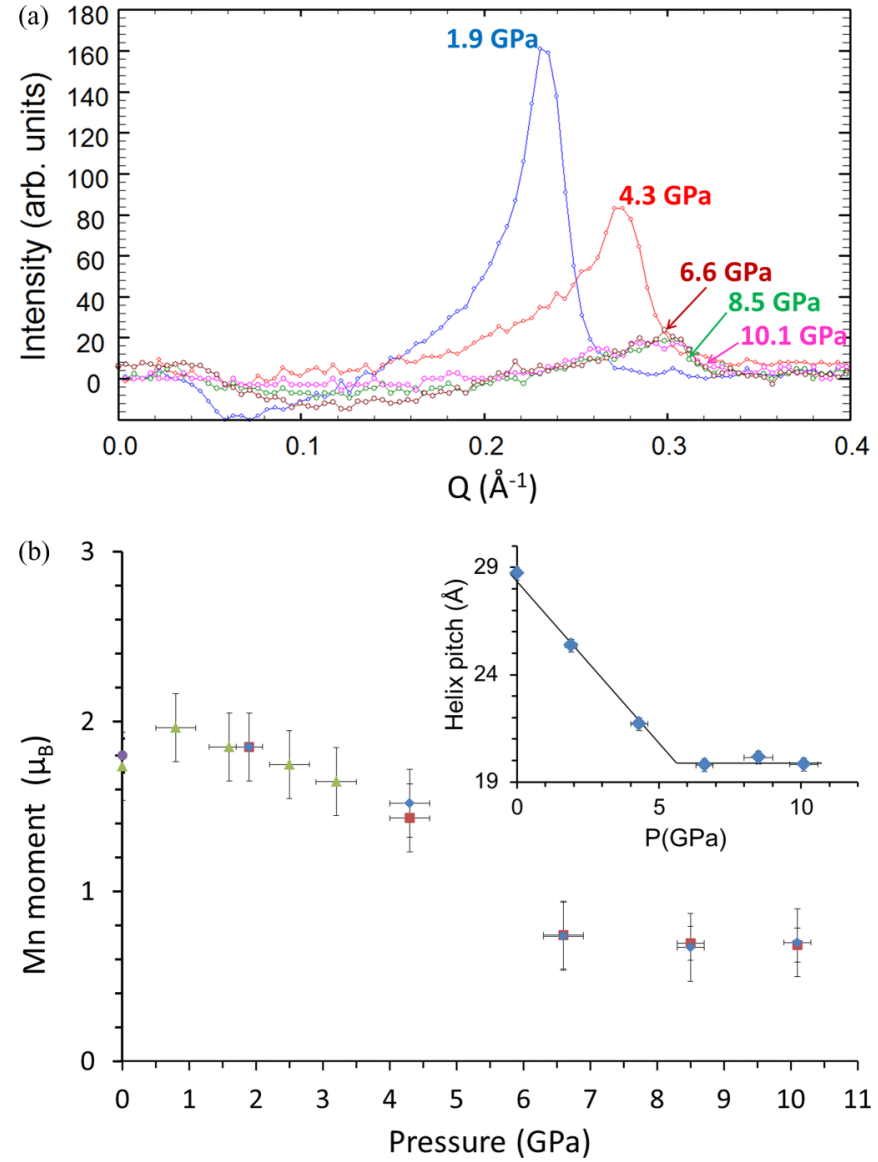

FIG. 3. (Color online) (a) Evolution of the zero satellite (scaled on nuclear peaks) measured well below $T_{N}$. (b) Evolution with pressure of the Mn saturated moment, measured well below $T_{N}$ (red squares: D20 refinement; blue lozenges: D20 scaled zero satellite intensity; green triangle: G6.1 scaled zero satellite intensity; purple circle; ambient pressure measurement). Inset of (b): Evolution of the helix pitch $(a / \zeta)$ with pressure.

value of $3 \mu_{B}$ that is expected for a $\mathrm{Mn}^{4+}$ ion. With increasing pressure $T_{N}$ decreases linearly with a slope of $-14 \mathrm{~K} / \mathrm{GPa}$, which is very similar to that of $\mathrm{MnSi}$, extrapolating to zero around $13 \mathrm{GPa}$ when the helical order is expected to collapse.

The particular behavior of MnGe can be construed based on the evolution of its magnetic ground state under compression, which we have investigated by density-functional theory (DFT) calculations (see the SM). A map of the ground-state energy versus lattice constant $a$ and spin moment $s$ was built using the fixed-spin technique. Figure 5(b) shows that a transition may occur from a HS state with a larger volume to a LS state with a reduced volume. The energy has a double-well shape and the metastable LS state can be reached by small (thermal) energies and/or compression. The theoretical bandstructure results have been refined by considering the effect of long-period spin fluctuations [22]. We employ a simple method to consider the spin fluctuation in the DFT approach [23]. In order to represent the band structure with a reduced magnetic moment, the exchange-correlation (XC) potential is rescaled by a single reduction factor $\xi$, which is specific for a particular state of a magnetic or paramagnetic system, and incorporates empirical information. Here, we used the

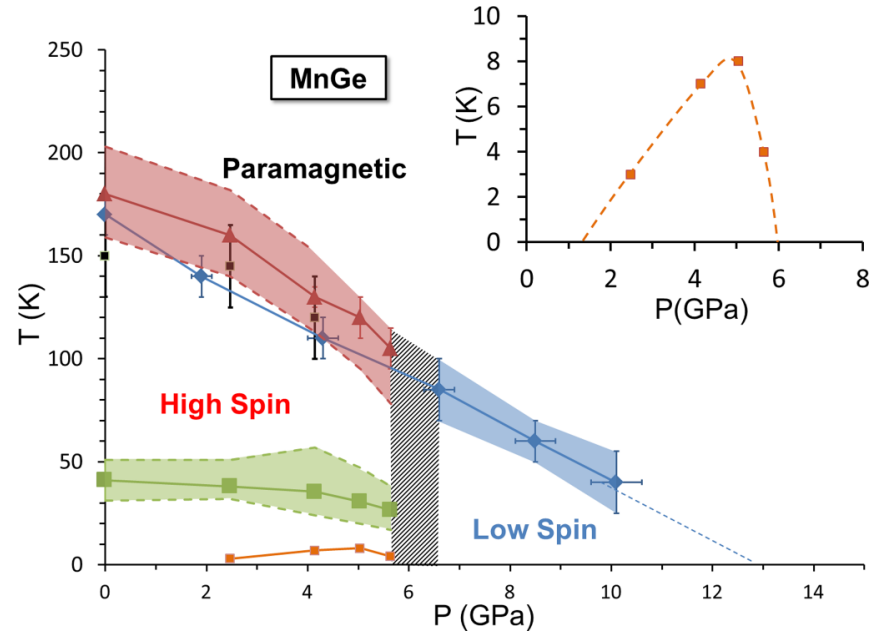

FIG. 4. (Color online) MnGe phase diagram. Pressure dependence of the transition temperatures. $T_{N}$ : neutrons (blue diamonds), susceptibility (red triangles), and resistivity (black squares); $T_{C}$ : susceptibility(green squares); $T_{M}$ : resistivity(orange squares). HS and LS correspond to high-spin and low-spin regions, respectively. Shaded regions are crossover regions deduced from the width of the transition. The inset shows the variation of $T_{M}$ with an enlarged scale.

measured magnetic moments in the HS and LS states [24] to improve the bare DFT results. A radical reorganization of the spin-polarized band structure is apparent between these two spin states, as shown in Fig. 5(a). The effect of SOC on the band structure is overall small. Hence, the HS-to-LS transition with volume reduction is a robust feature of the spin structure in $\mathrm{MnGe}$ and does not rely on the spin-relativistic effects. But in the HS state marked modifications appear: The small gap in the occupied part of the spin-majority band is smeared out and a van Hove-like singularity very close to the Fermi level is weakened. These modifications imply substantial differences in the DM exchange in the two different states, apart from their different spin moments. Calculating the cohesive properties under pressure, we find that the HS state is destabilized at a critical pressure, close to $P_{C} \simeq 6 \mathrm{GPa}$, and will be replaced by the LS state.

Figure 5(d) compares the theoretical equation of state (EOS) to the experimental one deduced from the D20 experiment at room temperature. The DFT calculations underestimate the equilibrium volume of the lattice cell in the HS state by about $2 \%$, which is within the usual accuracy of the method. The model predicts a further volume collapse by about $2 \%$ between the HS and LS state, which is not seen on the experimental EOS at $300 \mathrm{~K}$, or on our low temperature data points, but we note that the experimental EOS lies in between the two theoretical ones. This could be related to the detailed nature of the quantum transition, as discussed below. The experimental pressure dependence of the ordered Mn moment agrees quite well with the calculation [Fig. 5(c)] [24]. Especially, there is a good quantitative match of the critical pressure calculated for the HS-to-LS transition with the experimental value, situated around $6 \mathrm{GPa}$.

The pressure-temperature phase diagram in MnGe contrasts with those in MnSi [26] and FeGe [27], showing QPTs at critical pressures of 1.4 and $19 \mathrm{GPa}$, respectively. In $\mathrm{MnSi}$, 

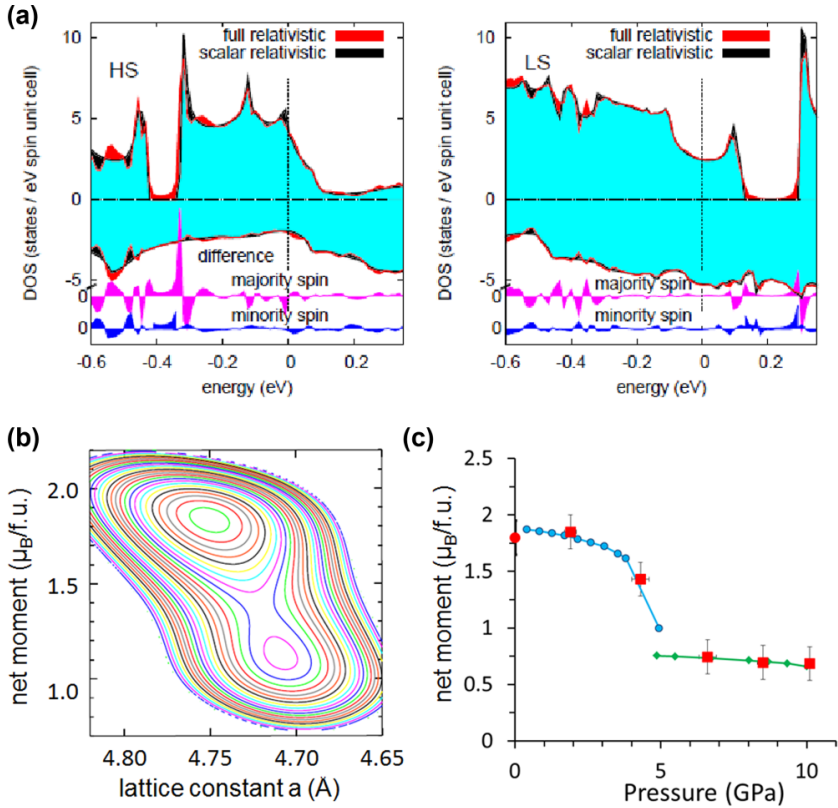

(d)

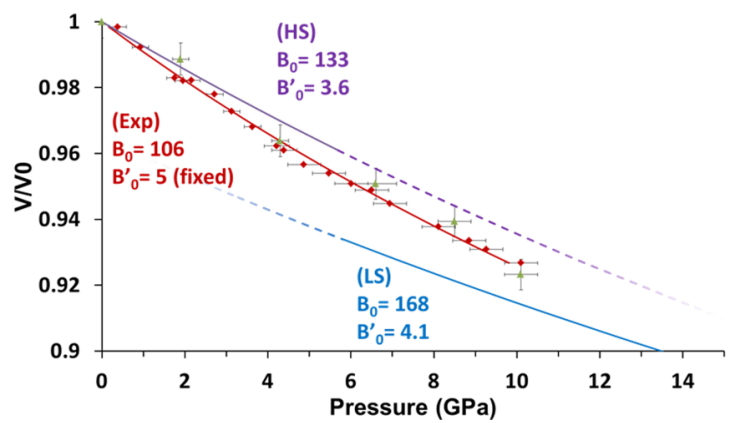

FIG. 5. (Color online) (a) Density of states (DOS) $\rho(E)$ for the high-spin state at equilibrium $a=4.755 \AA$ for the high-spin state (XC-reduction factor $\xi=0.915$ ), compared to the low-spin state at $a=4.700 \AA$ close to a critical pressure $p_{1}=6 \mathrm{GPa}$ with a stronger XC reduction, $\xi=0.720$. [The Fermi energy is at $E=0$, and $\rho(E)$ for the majority (minority) spin is counted positive (negative).] Results from scalar (sr) and full relativistic (fr-via approximate spin projection) calculations are compared in the main panel. The differences in the DOS, $\rho^{(\mathrm{fr})}-\rho^{(\mathrm{sr})}$, for majority and minority spins (lower part of the figures) are shown on the same scale. (b) Electron theoretical calculations on MnGe. Contour map of calculated total energy vs lattice constant and (fixed) spin $s$, and $\xi=0.915$. Energy levels are separated by $5 \mathrm{meV} /$ unit cell. (c) Calculated pressure dependence of the net moment per formula unit ( $\mu_{B} /$ f.u.) (blue HS state, green LS state) compared to experimental data (in red). (d) Equation of states for HS and LS states compared to experimental data (red points: room temperature measurement; green point: low temperature measurement). $B_{0}$ and $B_{0}^{\prime}$ are the Murnaghan parameters used to fit the EOS [25].

the QPT is of first order and magnetic moments persist in the NFL region just above the critical pressure (up to $\simeq 2 \mathrm{GPa}$ ). In FeGe, the collapse of spin polarization at very high pressure seems to be associated with a nearby insulating state [28]. In MnGe, the LS phase characterized by low constant $\mathrm{Mn}$ moments in a large pressure range above $P_{C}$ is specific to this compound. As the direct exchange and the Dzyaloshinskii-Moriya (DM) interaction originate in the same electronic structure, if the band structure remains essentially unchanged, the ratio of these two couplings, determining the helix pitch, should remain almost constant with temperature and pressure, as observed in $\mathrm{MnSi}$ and FeGe. However, in MnGe the helix pitch does not only show a very marked temperature and pressure dependence, it is also uncommonly short (about $30 \AA$ as compared with about $180 \AA$ in $\mathrm{MnSi}$ and $700 \AA$ in $\mathrm{FeGe}$ ), indicating a much stronger effect of the DM interaction than in other B20 magnets. The spin structure in $\mathrm{MnGe}$ is canted even over the lattice length scale, so that additional competing exchange couplings become relevant, recalling that different $\mathrm{Mn}-\mathrm{Mn}$ distances exist in the cubic cell. The ferromagnetic collinear order is frustrated and the helical twisting should depend both on the DM tensor and the competing neighbor exchange couplings in a subtle way, related to different orientations of the DM vector for different bonds [29]. The changes of the pitch may be caused both by the HS-to-LS transition and by variations of the competing magnetic interactions induced, as shown in other helical magnets [30,31].

According to the DFT results, the pressure-temperature phase diagram of $\mathrm{MnGe}$ should be governed by a discontinuous transition between the HS and LS states. We clearly observed two spin states in the phase diagram. The pressure-temperature phase diagram, thus, should be ruled either by an isolated critical point at zero temperature, or by a first-order line terminated by a critical end point (CEP) at nonzero temperature. This first-order line could be searched by $\mathrm{x}$-ray diffraction under high pressure and very low temperature. There is no evidence for a first-order structural transition in our neutron data. When atomic mobility allows it, a HS-to-LS transition may result in chemical phase segregation, as shown in Laves hydrides [32,33]. In MnGe, owing to necessary volume strain between the HS and LS state, a coexistence of these two states in spatially separated domains cannot be realized. Rather, an intermediate magnetoelastic state with strong spin fluctuations is expected, as in other metallic systems with a spin-state instability such as Invar alloys [34,35], and the spin-state transition takes place by a smooth crossover.

We thank C. Lacroix, P. Bonville, S. M. Stishov, and S. V. Grigoriev for useful discussions. The postdoctoral training of M.D. is funded by the Triangle de la Physique. The stays of O.L.M. at LLB in 2012-2013 were funded by the Triangle de la Physique and Labex Palm-Emergence. A.V.T. acknowledges the support of the Russian Foundation for Basic Research (Grant No. 14-02-00001).
[1] J. Thompson, Z. Fisk, and G. Lonzarich, Physica B 161, 317 (1989).
[2] N. Manyala, Y. Sidis, J. DiTusa, G. Aeppli, D. Young, and Z. Fisk, Nature (London) 404, 581 (2000). 
[3] C. Pfleiderer, D. Reznik, L. Pintschovius, H. v. Lohneysen, M. Garst, and A. Rosch, Nature (London) 427, 227 (2004).

[4] X. Z. Yu, Y. Onose, N. Kanazawa, J. H. Park, J. H. Han, Y. Matsui, N. Nagaosa, and Y. Tokura, Nature (London) 465 , 901 (2010).

[5] N. Romming, C. Hanneken, M. Menzel, J. E. Bickel, B. Wolter, K. von Bergmann, A. Kubetzka, and R. Wiesendanger, Science 341, 636 (2013).

[6] A. Neubauer, C. Pfleiderer, B. Binz, A. Rosch, R. Ritz, P. G. Niklowitz, and P. Böni, Phys. Rev. Lett. 102, 186602 (2009).

[7] N. Nagaosa and Y. Tokura, Nat. Nanotechnol. 8, 899 (2013).

[8] M. Lee, W. Kang, Y. Onose, Y. Tokura, and N. P. Ong, Phys. Rev. Lett. 102, 186601 (2009).

[9] R. Ritz, M. Halder, C. Franz, A. Bauer, M. Wagner, R. Bamler, A. Rosch, and C. Pfleiderer, Phys. Rev. B 87, 134424 (2013).

[10] C. Pfleiderer and U. K. Roßler, Nature (London) 447, 157 (2007).

[11] S. V. Grigoriev et al., Phys. Rev. Lett. 110, 207201 (2013).

[12] K. Shibata, Z. X. Yu, T. Hara, D. Morikawa, N. Kanazawa, K. Kimoto, S. Ishiwata, Y. Matsui, and Y. Tokura, Nat. Nanotechnol. 8, 723 (2013).

[13] H. Takizawa, T. Sato, T. Endo, and M. Shimada, J. Solid State Chem. 73, 40 (1988).

[14] N. Kanazawa, Y. Onose, T. Arima, D. Okuyama, K. Ohoyama, S. Wakimoto, K. Kakurai, S. Ishiwata, and Y. Tokura, Phys. Rev. Lett. 106, 156603 (2011).

[15] Y. Shiomi, N. Kanazawa, K. Shibata, Y. Onose, and Y. Tokura, Phys. Rev. B 88, 064409 (2013).

[16] J. Y. Choi, S. Kang, S. W. Seo, W. J. Kwon, and Y. I. Shin, Phys. Rev. Lett. 111, 245301 (2013).

[17] O. L. Makarova, A. V. Tsvyashchenko, G. Andre, F. Porcher, L. N. Fomicheva, N. Rey, and I. Mirebeau, Phys. Rev. B 85, 205205 (2012).

[18] N. Kanazawa, J. H. Kim, D. S. Inosov, J. S. White, N. Egetenmeyer, J. L. Gavilano, S. Ishiwata, Y. Onose, T. Arima, B. Keimer, and Y. Tokura, Phys. Rev. B 86, 134425 (2012).

[19] U. K. Rößler, J. Phys.: Conf. Ser. 391, 012104 (2012).
[20] See Supplemental Material at http://link.aps.org/supplemental/ 10.1103/PhysRevB.89.180407 for a description of the sample synthesis and experimental techniques, a detailed temperature dependence of the neutron data at ambient and under pressure, and a full description of the ab initio calculations.

[21] A. Tsvyashchenko et al., Solid State Phenom. 190, 225 (2012).

[22] T. Moriya, Spin Fluctuations in Itinerant Electron Magnetism, Springer Series in Solid-State Sciences Vol. 56 (Springer, Berlin, Heidelberg, 1985)

[23] L. Ortenzi, I. I. Mazin, P. Blaha, and L. Boeri, Phys. Rev. B 86, 064437 (2012).

[24] The calculation in the HS state with $\xi=0.915$ predicts a magnetic moment of $2.10 \mu_{B}$ on a Mn site and a small antiferromagnetic moment of $-0.25 \mu_{B}$ on a Ge site, yielding a total moment of $1.85 \mu_{B} /$ f.u. at ambient pressure. For the LS state, a net moment of $0.73 \mu_{B}$ /f.u. was adjusted with $\xi=0.720$ at a volume corresponding to pressure $6.7 \mathrm{GPa}$. The experimental data do not allow one to refine a moment on $\mathrm{Ge}$, so that all the spin density is assumed to be carried by Mn .

[25] F. D. Murnaghan, Proc. Natl. Acad. Sci. U.S.A. 30, 244 (1944)

[26] C. Pfleiderer, P. Böni, T. Keller, U. K. Rößler, and A. Rosch, Science 316, 1871 (2007).

[27] P. Pedrazzini, H. Wilhelm, D. Jaccard, T. Jarlborg, M. Schmidt, M. Hanfland, L. Akselrud, H. Q. Yuan, U. Schwarz, Y. Grin, and F. Steglich, Phys. Rev. Lett. 98, 047204 (2007).

[28] M. Neef, K. Doll, and G. Zwicknagl, Phys. Rev. B 80, 035122 (2009).

[29] V. A. Chizhikov and V. E. Dmitrienko, Phys. Rev. B 85, 014421 (2012).

[30] I. Mirebeau, I. N. Goncharenko, and I. V. Golosovsky, Phys. Rev. B 64, 140401 (2001).

[31] O. L. Makarova, I. Mirebeau, S. E. Kichanov, J. RodriguezCarvajal, and A. Forget, Phys. Rev. B 84, 020408 (2011).

[32] I. N. Goncharenko, P. Cadavez-Peres, I. Mirebeau, and O. L. Makarova, Europhys. Lett. 54, 807 (2001).

[33] I. N. Goncharenko, P. Cadavez-Peres, I. Mirebeau, O. L. Makarova, T. Le Bihan, and M. Mezouar, Phys. Rev. B 68, 214418 (2003).

[34] T. Moriya and K. Usami, Solid State Commun. 34, 95 (1980).

[35] M. van Schilfgaarde, I. Abrikosov, and B. Johansson, Nature (London) 400, 46 (1999). 